\title{
Anti Iglon5 Syndrome: What we know so far? A non-systematic review
}

\section{Caroline Figueiredo da Silva ${ }^{1}$; Gustavo Figueiredo da Silva ${ }^{1}$; Washigton Luiz Gomes de Medeiros Junior'; Marcus Vinícius Magno Gonçalves²}

1- Medical student - Department of Medicine, University of the Region of Joinville (UNIVILLE), Brazil.

2 - Medical Doctor, PhD and Professor of Neurology, University of the Region of Joinville (UNIVILLE), Brazil.

\section{Corresponding Author}

Caroline Figueiredo da Silva

Department of Medicine - University of the Region of Joinville. Paulo Malschitzki, 10 - Zona Industrial Norte, CEP 89201-972, Joinville, Santa Catarina, Brazil. Phone number: +55 419 9927-8674. E-mail: caroolfiga@gmail.com

\begin{abstract}
The objective of this review is to do an overview about the current knowledge of Anti Iglon5 Syndrome, a disease that was first described in 2014. The IgLON proteins are a family of cell adhesion molecules and the presence of antibodies against IgLON5 are crucial for diagnosis of Anti IgLON5 Syndrome. This syndrome has an expanded clinical spectrum that involves prominent sleep disorder, progressive bulbar dysfunction, gait instability with abnormal eye movements reminiscent and cognitive deterioration sometimes associated with chorea. The main neuropathological finding is the neuronal loss with hyperphosphorylated tau (p-Tau) protein accumulation at hypothalamus, brainstem tegmentum, hippocampus, periaqueductal gray matter, medulla oblongata and upper cervical cord. The exact pathogenesis is still unclear and involves a neurodegenerative process and autoimmune response. The early diagnosis is
\end{abstract}


important to avoid unnecessary tests and prevent complications. Important resources for diagnosis are the antibody testing of serum and CSF for IgLON5-IgG. The mortality of anti IgLON5 syndrome is high and new studies published described a good response to immune therapy. However, the response to immune therapy depends of some clinical and analytical characteristic. In addition, future studies are needed to thoroughly study the aspects of pathogenesis and treatment of this important pathological syndrome.

\section{Keywords}

Anti-IgLON5 disease, sleep disorders, tau protein accumulation, Immunotherapy, Neurodegenerative Diseases.

\section{Abbreviations}

CNS - Central Nervous System

CSF - Cerebrospinal Fluid

IgG - Immunoglobulin G

MRI - Magnetic Resonance Imaging

PSP-like - Progressive Supranuclear Palsy

p-Tau - Hyperphosphorylated Tau Protein

REM - Rapid Eye Movement

\section{MANUSCRIPT}

\section{Methods:}

This study is a non-systematic review with based on articles published since 2014 in Medline accessed at Pubmed. The discussion divided in points to give an overview about the 
pathophysiology, clinical manifestations, diagnoses, and treatment of the Anti- IgLON5 syndrome.

\section{Discussion:}

\section{$\underline{\text { Background }}$}

The first reported of Anti-IgLON5 syndrome was in 2014, when antibodies against the cell surface protein IgLON5 were described associated with a complex neurological syndrome of sleep disturbances and movement disorders. Anti-IgLON5 antibodies have a prevalence of 12 in 150,000 patients per year, however considering the unreported patients, the prevalence can might be much higher ${ }^{1}$. Since 2014 , news research showed an expanded clinical spectrum that involves sleep dysfunction, bulbar dysfunction, chorea and progressive supranuclear palsy-like symptoms whereas dysautonomia and cognitive decline are presents in more advanced stages ${ }^{2}$. The objective of this paper is to review the main finds about Anti IgLON5 syndrome.

\section{Clinical manifestations}

The onset of anti-IgLON5 disease is insidious and may be between 45 to 75 years ${ }^{3}$. Moreover, the onset involves progressive sleep and brainstem disorders. Whereas neuropsychiatric disorders, dysautonomia and hyperexcitability (myoclonus, cramps, and exaggerated startle) are most commonly described in late stages ${ }^{3,4}$. Most often, the sleep disorder involves, during non-REM (Rapid Eye Movement) and REM, sleep breathing changes and parasomnias while, among the brainstem disorders, gait abnormalities, dysphagia, dysarthria and oculomotor problems ${ }^{3,5}$. Furthermore, there are described cognitive impairment and psychiatric symptoms in the neuropsychiatric field and myoclonus, cramps and exaggerated startle as hyperexcitability symptoms ${ }^{3}$.

Thus, four clinical presentations were characterized according to predominant symptoms: a sleep disorder with parasomnia and sleep breathing difficulty, a bulbar syndrome including 
dysphagia, sialorrhea, stridor, or acute respiratory insufficiency, a syndrome resembling progressive supranuclear palsy (PSP-like) (vertical and horizontal supranuclear gaze palsies) and cognitive decline with or without chorea ${ }^{6}$ (figure 1 ).

In addition, despite the evidence that anti-IgLON5 disease is related to central nervous system, clinical descriptions suggest that there is an important role of anti-IgLON5 antibodies on peripheral nerves. Thus, peripheral involvement with neuromyotonic discharges, muscle weakness and wasting may be related to anti-IgLON5 disease also ${ }^{1}$.

Understanding anti-IgLON5 syndrome: The pathophysiology

The IgLON proteins are a family of cell adhesions molecules, containing five different proteins (IgLON 1-5). Structurally IgLONs are about 340 amino acids long and tied to the cell membrane. Until today, the pathophysiological function of IgLON5 is unknown ${ }^{7,8}$. It has been suggested that IgLONs play a role in the evolution of brain anatomy and complex maturation, influencing cellular migration and brain-blood barrier integrity ${ }^{9.10}$.

In addition, antibodies against IgLON5 are crucial for diagnosis and are present in serum and in almost all cases in CSF, being strongly associated with the HLA-DRB1*1001 and HLADQB1*0501 alleles. Patients with anti-IgLON5 disease display all four isotypes of Immunoglobulin $\mathrm{G}(\mathrm{IgG})$ but predominantly $\mathrm{IgG} 1$ (94\%) and $\mathrm{IgG} 4$ (92\%), with a higher density of IgG4 than IgG1 in serum ${ }^{6,10}$.

The main neuropathological finding in anti-IgLON5 disease is the neuronal loss with hyperphosphorylated tau (p-Tau) protein accumulation (3-repeat and 4-repeat isoform). Hypothalamus and brainstem tegmentum were the sites of $\mathrm{p}$-Tau protein greatest aggregation, still with a rostrocaudal gradient involving the hippocampus, periaqueductal gray matter, medulla oblongata, and upper cervical cord. No inflammatory infiltrates or concomitant abnormal protein deposits of beta-amyloid or alpha-synuclein were detected ${ }^{5,11}$. In contrast, 
substantia nigra, basal ganglia, cortex, thalamus and cerebellum are preserved or slightly affected, showing a different anatomical brain distribution than primary taupathies (e.g., corticobasal syndrome, progressive supranuclear palsy) ${ }^{12}$.

An important study conducted by Gelpi et al. ${ }^{5}$ suggested research criteria for the neuropathological diagnosis of anti IgLON5 disease. In addition to the neuropathological findings, additional clinical and immunological criteria were used to classify the findings as "definitive", "probable" or "possible" diagnosis. Thus, it was established that a diagnosis is 1) "definitive" when the neuropathological pattern is present with antibodies detection, regardless of CSF or serum; 2) "probable" when neuropathological findings are present, along with clinical history or alleles confirmation (HLA-DRB1*1001 and HLA-DQB1*0501), even with unknown antibody status; and 3) "possible" when neuropathological findings are present without immunological or clinical information ${ }^{5}$.

The exact pathogenesis of this disorder is still unclear and a primary neurodegenerative process can trigger the autoimmune response. According to Gaig et ll $^{6}$., the antibody interference of the IgLON5 interaction with the internal cytoskeletal network, generating neuronal dysfunction and subsequently, neurodegeneration, may explain the accumulation of neuronal $\mathrm{p}$-Tau ${ }^{6}$.

Key points for diagnosis:

Based in those symptoms, adults with unexplained sleep disorder, brainstem disorders, dysautonomia, hyperexcitability and neuropsychiatric disorders should be screened for IgLON5 disease. The early diagnosis is important to avoid unnecessary tests, prevent complications, as acute respiratory failure and irreversible neuronal damage, and have the potential to improve outcome with the correct treatment ${ }^{6,13}$.

For screening, antibody testing of serum and CSF for IgLON5-IgG is crucial ${ }^{8}$. Besides, HLADRB1*1001 and HLA-DQB1*0501 are strongly associated to presence of anti-IgLON5 
antibodies. In a review of reported cases, Nissen MS and colleague $2019^{10}$ demonstrated that antibodies, in serum and CSF, are positive in 95 and 100\% of cases, respectively. Moreover, was demonstrated that brain FDG-PET CT could be more sensitive than Magnetic Resonance Imaging (MRI), was abnormal in 50\% of cases, and abnormal polysomnography was found in $95 \%$ of cases ${ }^{10}$.

Additional paraclinical investigations, that may be genetic clues to an autoimmune diagnosis, such as subacute presentation, history of autoimmune disease, electromyography, an inflammatory CSF or inflammatory-appearing brain imaging, are infrequent and nonspecific. Other tests may be important according to the clinical presentation ${ }^{4,8}$.

Since the symptoms are nonspecific and wide, it is important to perform differential diagnosis with other diseases. Among the diseases, progressive supranuclear palsy, others tauopathy, Central Nervous System (CNS) Whipple disease and multiple system atrophy are some examples ${ }^{8,14}$.

\section{Treatment Resources:}

Anti IgLON5 syndrome is a controversial disease where the answer for the pathological mechanism is uncertain. This syndrome can be considered as degenerative disease with a secondary inflammatory response against IgLON5 or an autoimmune disorder that the neuronal accumulation of tau mediated by antibodies results in a functional disorder followed by neurodegeneration $^{15}$. The mortality of anti IgLON5 syndrome is not accurate, however a review with 58 cases found a mortality rate of $34 \%$ and no association between the deaths and the response to the treatment ${ }^{10}$. Additionally, the literature shows only one case of spontaneous improvement ${ }^{15}$.

In the past, a very low response to immunotherapy was reported in the first cases. However, new studies have been published describing a good response to immunotherapy what can 
possible be explained by the included of patients with non-classical phenotypes. Also, a higher response rate in patients with cognitive impairment and non-classical phenotypes happened in patients without HLA-DRB*1001 and cerebral spinal fluid inflammation. A systematic review showed that combination therapy can be more effective that monotherapy. Monotherapy response to immunotherapy was describe in $7 / 22$ patients $(31,8 \%)$ while with combination therapy there was a response in $14 / 21(66,6 \%)^{15}$.

The possibly benefits of an immediate immunotherapy is an early interruption of antibody production results to a delay or stop of the pathomechanism and a reduction of IgLON5 antibodies ${ }^{8}$. Although, the reason why some patients are more responsive to immunotherapy is not clear and possible have relations with the different phenotypes ${ }^{16}$.

\section{Conclusions}

Therefore, the anti-IgLON5 syndrome is a disease with high mortality that has several clinical manifestations. Most often, the sleep disorders involve, during REM and non-REM sleep, sleep breathing changes, parasomnias, brain stem disorders, gait abnormalities, dysphagia, dysarthria, and oculomotor problems. The pathophysiology of this condition is still unclear but it is known there is an IgLON5 antibody involvement (an important biomarker of the disease) and there is hyperphosphorylated Tau protein accumulation mainly in the hypothalamus and brain stem tegmentum. The analysis of symptoms, serum and CSF samples, alleles (HLADRB $1 * 1001$ and HLA-DQB $1 * 0501)$, and the p-Tau protein presence allows the patient to be categorized as "definitive", "probable" and "possible" diagnosis. In addition, patients with anti-IgLON5 disease may respond to immunotherapy, but this depends on some clinical and analytical characteristics. Finally, future studies are needed to thoroughly study each particularity of this important pathological syndrome.

\section{Summary}




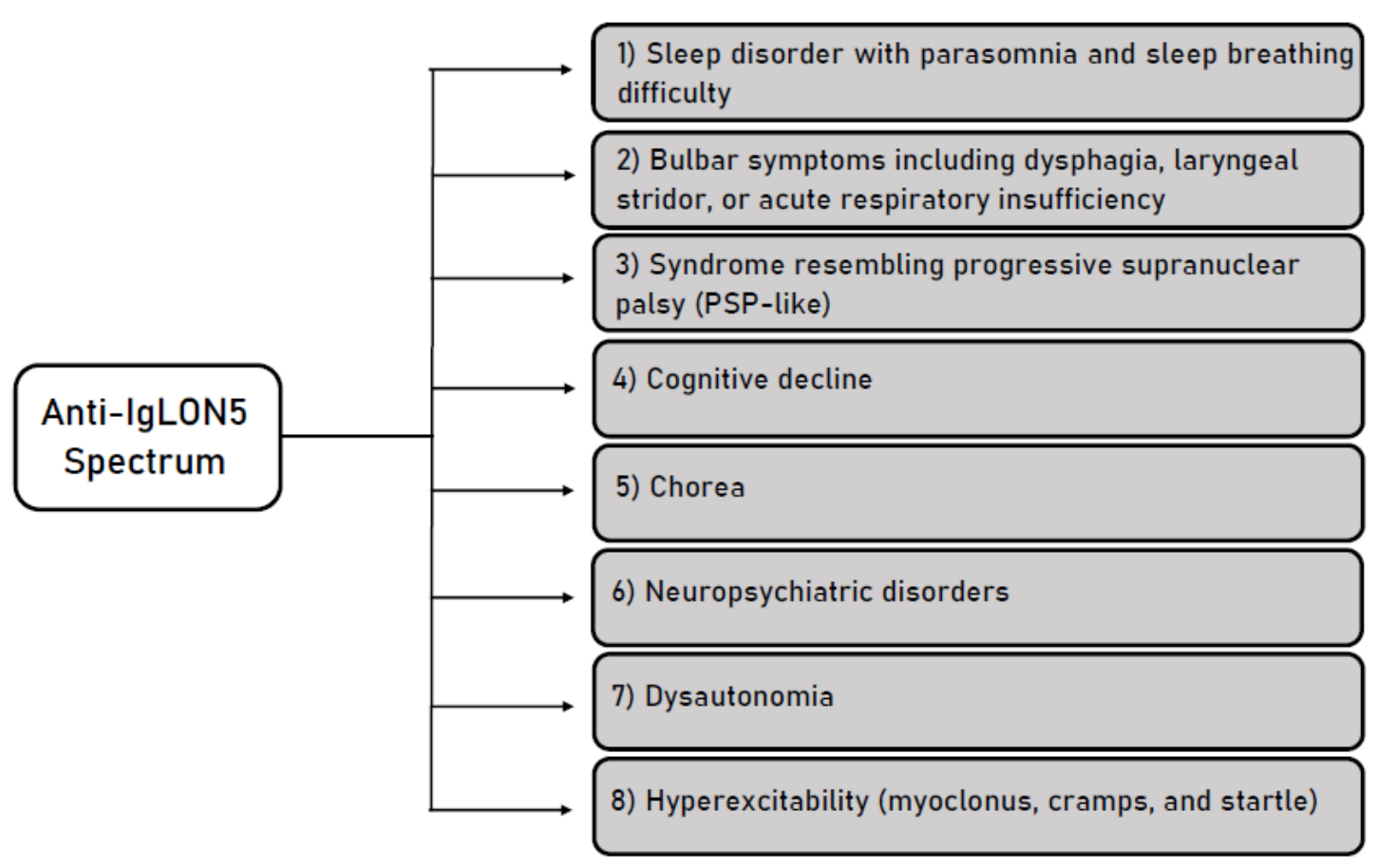

Figure 1: Summary of Anti- IgLON5 Spectrum of clinical manifestations.

\section{Acknowledgement:}

No targeted funding reported.

\section{Disclosure}

The authors report no disclosures. All authors and contributors agree to the conditions outlined in the Authorship and Contributorship section of the Information for Author. The authors have read the Journal's position on issues involved in ethical publication. There was no sponsorship for the scientific article and there was no conflict of interest with all the authors. 


\section{REFERENCES:}

1 Wenninger S. Expanding the Clinical Spectrum of IgLON5-Syndrome. Journal of Neuromuscular Diseases. 2017;4(4):337-339.

${ }^{2}$ Montagna et al. IgLOn5- Associated Encephalitis with atypical brain magnetic resonance imaging and cerebrospinal fluid changes. Frontiers in Neurology. 2018;9: 329.

${ }^{3}$ Honorat $\mathrm{J}$ et al. IgLON5 antibody- Neurological accompaniments and outcomes in 20 patients. Neurology - Neuroimmunology Neuroinflammation. 2017;4(5): e385.

${ }^{4}$ Schröder $\mathbf{J}$ et al. Isolated dysphagia as initial sign of anti-IgLON5 syndrome. Neurology Neuroimmunology Neuroinflammation. 2016;4(1) :e302.

5 Gelpi E et al. Neuropathological criteria of anti-IgLON5-related tauopathy. Acta Neuropathologica. 2016;132(4):531-543.

6 Gaig $\mathrm{C}$ et al. Clinical manifestations of the anti-IgLON5 disease. Neurology. 2017;88(18):1736-1743.

${ }^{7}$ Karagogeos D. Neural GPI-anchored cell adhesionmolecules. Front Biosci. 2003;8:s130420.

${ }^{8}$ Heidbreder, P. Anti- Ig LON5 Disease. Curr Treat Option Neurol. 2018; 20(8):29.

${ }^{9}$ Kubick N, Brosamle D, Mickael ME. Molecular evolution and functional divergence of the IgLON family. Evol Bioinformatics Online. 2018: 21;14:1176934318775081.

${ }^{10}$ Nissen MS, Blaabjerg M. Anti-IgLON5 Disease : A Case With 11-Year Clinical Course and Review of the Literature 2019;10:1-7. 
${ }^{11}$ Sabater L et al. A novel non-rapid-eye movement and rapid-eye-movement parasomnia with sleep breathing disorder associated with antibodies to IgLON5: a case series, characterisation of the antigen, and post-mortem study. Lancet Neurol 2014;13:575-586.

${ }^{12}$ Iranzo A. Parasomnias and sleep-related movement disorders in older adults. Sleep Med Clin. 2018;13(1):51-61.

${ }^{13}$ Graus F and Santamaría J. Understanding anti-IgLON5 disease. Neurol Neuroimmunol Neuroinflamm 2017;4:e393

14. Morales-Briceño H et al. IgLON5-mediated neurodegeneration is a differential diagnosis of CNS Whipple disease. Neurology. 2018;90(24):1113-1115.

15 GARCIA et al. Response to immunotherapy in anti-IgLON5 disease: a systematic review. Acta Neurol Scand. 2020: 1(4):263-270.

16 LOGMIN et al. Intravenous immunoglobulins as first-line therapy for IgLON5 encephalopathy. 2019. https://doi.org/10.1007/s00415-019-09221-3 
